\title{
Intussusception Induced by Burkitt Lymphoma in an Adult Patient
}

\author{
Olivia Makenzie Boyette ${ }^{1}$, Bradley Casey III $^{2}$, Stephanie Khadeejah Tom ${ }^{3}$, Todd Nicholas Tom ${ }^{4}$ \\ ${ }^{1}$ Kentucky College of Osteopathic Medicine, University of Pikeville, Pikeville, United States of America \\ ${ }^{2}$ Ross University School of Medicine, Ross University, Bridgetown, Barbados \\ ${ }^{3}$ University of Louisville School of Medicine, University of Louisville, Louisville, United States of America \\ ${ }^{4}$ Department of General Surgery, Tug Valley ARH Regional Medical Center, South Williamson, United States of America
}

Email address:

ttom@arh.org (T. N. Tom)

\section{To cite this article:}

Olivia Makenzie Boyette, Bradley Casey III, Stephanie Khadeejah Tom, Todd Nicholas Tom. Intussusception Induced by Burkitt Lymphoma in an Adult Patient. Journal of Surgery. Vol. 8, No. 2, 2020, pp. 67-70. doi: 10.11648/j.js.20200802.15

Received: January 30, 2020; Accepted: March 31, 2020; Published: April 14, 2020

\begin{abstract}
Intussusception is defined as the telescoping of an intestinal region into an adjacent region of intestine. We present an interesting case of a retrograde intussusception due to an underlying Burkitt lymphoma. Case Presentation: We have a 41-year-old Caucasian male who presented to the Emergency Department (ED) with complaints of left sided periumbilical abdominal pain that began several months prior. Computed tomography (CT) scan of the abdomen and pelvis with oral and IV contrast showed a colonic mass in the region of the ileocecal valve. Patient denied any fever, vomiting, melena, or hematochezia. He was scheduled for a colonoscopy with surgical services. Upon colonoscopy, intussusception in the region of the ileocecal valve was discovered and cecectomy was scheduled for the same day. Intussusception was removed via hand assisted laparoscopic cecectomy with functional end-to-end anastomoses. Gross dissection of the specimen post cecectomy revealed a $5 \mathrm{~cm}$ by $5 \mathrm{~cm}$ mass as the lead point for intussusception (Figures 4,5 ). The mass was sent for pathology which revealed Burkitt lymphoma. Conclusion: Intussusception is a rare cause of abdominal pain in adults but should be considered because it may be an indicator of underlying malignancy. Unlike intussusception in the pediatric patient, which can be managed non-operatively, treatment in adults is usually surgery.
\end{abstract}

Keywords: Colon Cancer, Burkitt Lymphoma, Colon Surgery, Colorectal Surgery, Abdominal Pain, Hand Assisted Laparoscopic Cecetomy, Ileocecal Valve, Colonoscopy, Intestinal Obstruction, Intussusception

\section{Introduction}

Intussusception is defined as the telescoping of an intestinal region into an adjacent region of intestine [1]. In adults, intussusception is typically due to formation of a lead point within the bowel itself which gets pulled forward by normal peristalsis of the bowel. This causes the bowel adjacent to the lead point to prolapse over the affected segment of bowel. It is estimated that only $5 \%$ of all intussusceptions occur in adults and approximately $5 \%$ of bowel obstructions in adults are the result of intussusception [2]. In contrast to pediatric patients, $90 \%$ of the time in adults, it is caused by well-established pathologic mechanisms, such as carcinomas. Small bowel intussusceptions are more frequent, but colonic intussusceptions may be caused by malignant neoplasms [3].
The diagnosis of intussusception in an adult is difficult due to varying presentations [4]. Intussusception presents clinically with intermittent, colicky abdominal pain, accompanied with nausea and vomiting [1]. To date, only a select few studies have made a connection between intussusception and lymphomas. We present an interesting case of a retrograde intussusception due to an underlying Burkitt lymphoma.

\section{Presentation of Case}

A 41-year-old Caucasian male presented to the Emergency Department (ED) with complaints of left sided periumbilical 
abdominal pain that began months prior. The pain was described as achy, constant and non-radiating in nature. The patient stated his pain was worse after eating but was not able to identify which foods worsened his pain. Patient denied fever, vomiting, melena, or hematochezia. He reported associated nausea, constipation with intermittent diarrhea and decrease in appetite. He also endorsed a recent unintentional $10 \mathrm{lb}$ weight loss over the past several months. His last bowel movement was one week prior to presentation. He was not taking any medications at the time of presentation. Patient had a 46 pack year history of tobacco smoking and denied any alcohol or recreational drug use. Surgical history included appendectomy.

Upon arriving to the ED, his vitals were within normal limits. He appeared in obvious discomfort. Abdominal exam revealed diffuse tenderness to palpation, worse in the periumbilical region, without guarding or rebound tenderness. Hemoglobin was 12.6 (Normal 13.1-16.0 g/dL) and Hematocrit 37.4 (Normal 39.6 - 48.7\%). All other labs were within normal limits including the CMP, lactate and the remainder of the $\mathrm{CBC}$.

Computed tomography (CT) scan of the abdomen and pelvis with oral and IV contrast showed an incomplete distal small bowel obstruction due to a $5.5 \mathrm{~cm} \times 5.0 \mathrm{~cm}$ mass at the ileocecal junction (Figures 1, 2, 3). Metallic surgical clips were noted in the region of the cecum, likely related to prior appendectomy. Several small, benign-appearing, low-attenuation areas in the liver are likely small cysts. At this point, general surgery was consulted to see the patient in the ED. Upon interview in the ED, patient was found to be hemodynamically stable and the decision was made to send the patient home and schedule colonoscopy for the following day.

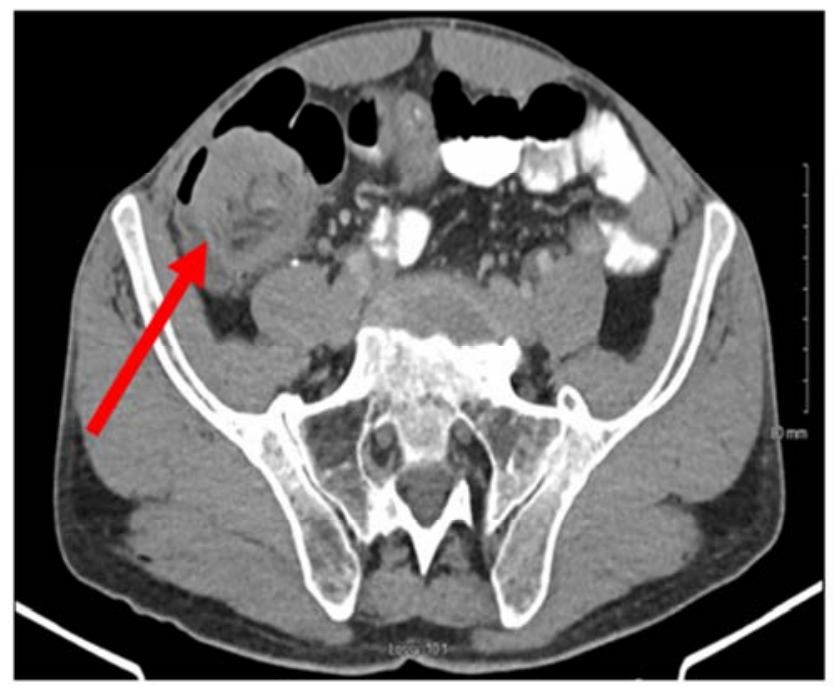

Figure 1. Transverse computed tomography scan of the abdomen and pelvis with oral and intravenous contrast.

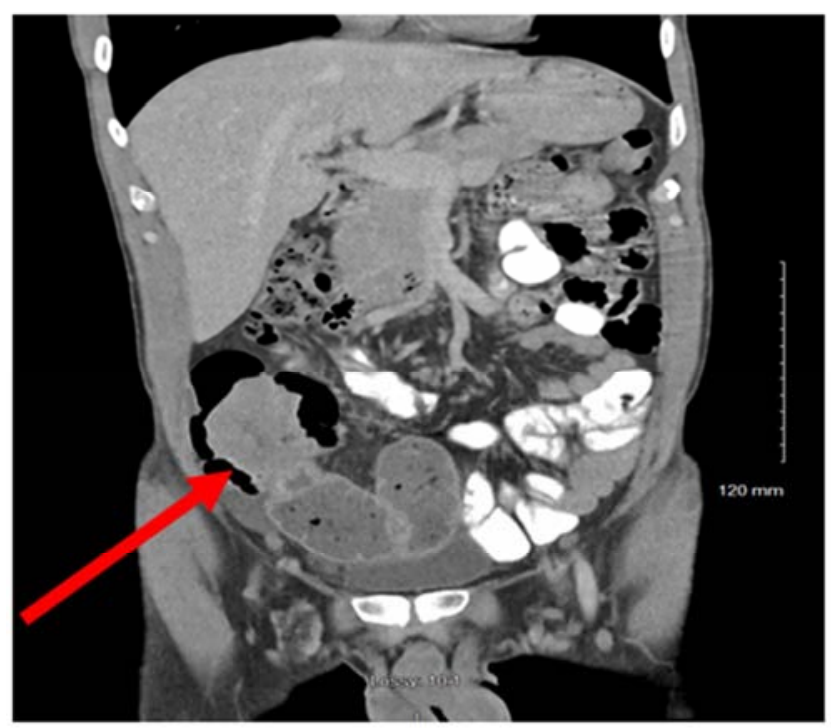

Figure 2. Coronal computed tomography scan of the abdomen and pelvis with oral and intravenous contrast.

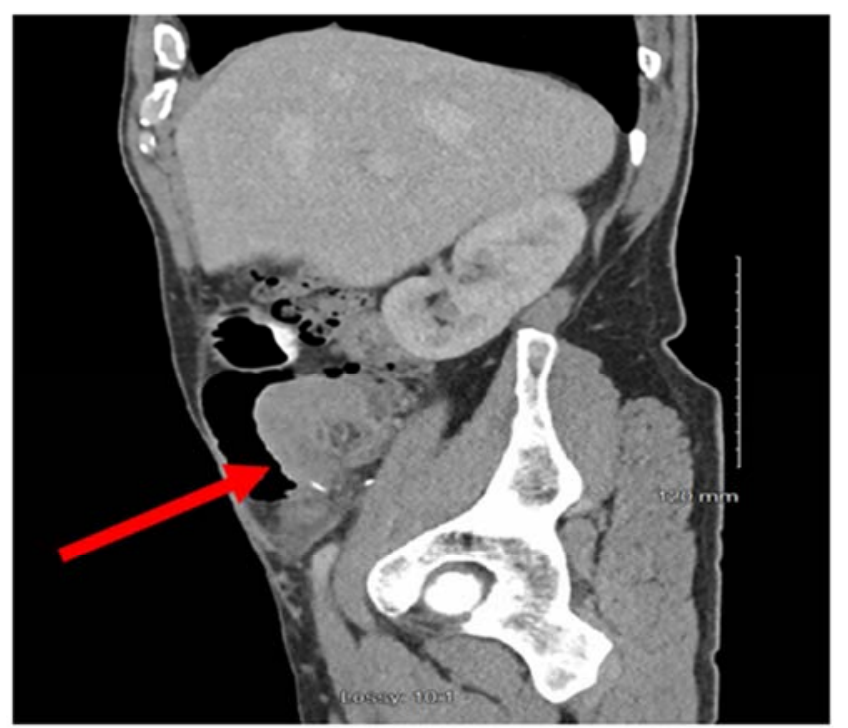

Figure 3. Sagittal computed tomography scan of the abdomen and pelvis with oral and intravenous contrast.

\section{Therapeutic Interventions}

Patient was scheduled for colonoscopy with possible biopsy for the following day. Upon colonoscopy, we discovered intussusception in the region of the ileocecal valve with evidence of necrosis on the inverted surface, with greater than $90 \%$ occlusion of the bowel. Cecectomy was scheduled for the same day.

Intussusception was removed via hand assisted laparoscopic cecectomy with functional end-to-end anastomoses performed. A $5 \mathrm{~cm}$ umbilical incision was made for specimen extraction. After the cecum was mobilized medially, the cecum and terminal ileum were delivered into the midline incision. The patient was found to have a retrograde intussusception with the cecum overlying the ileum. 
Gross dissection of the specimen post cecectomy revealed a large mass as the lead point for intussusception (Figures 4, 5). The mass was sent for pathology which revealed Epstein-Barr virus (EBV) positive Burkitt lymphoma. The pathologist's gross description of the tumor is as follows: there is an ovoid elevated tumor mass measuring $6.5 \times 5.5 \times 2 \mathrm{~cm}$, situated $2.5 \mathrm{~cm}$ proximal to the ileocecal valve. The proximal edge of the mass is $2.8 \mathrm{~cm}$ from the proximal margin. Cut surface of the tumor mass is tan-white in color, firm, and smooth. The tumor appears to involve the entire thickness of the bowel wall. Eleven lymph nodes are dissected from the attached adipose tissue, the largest measuring $0.4 \mathrm{~cm}$ in greatest dimension.

Patient was admitted following surgery and placed on bowel rest. He was discharged home after an uneventful three day hospital course. Patient followed up in office on post-op day seven and reported complete resolution of abdominal pain and constipation. Staples were removed during the follow-up visit and the post-operative decision was made to consult oncology to discuss the patient's course of treatment for Burkitt lymphoma.

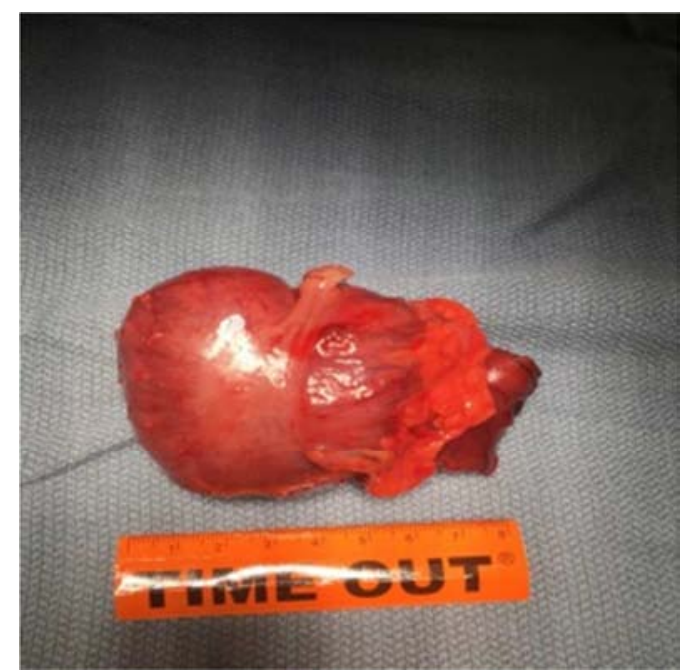

Figure 4. Gross specimen of intussusception prior to dissection.

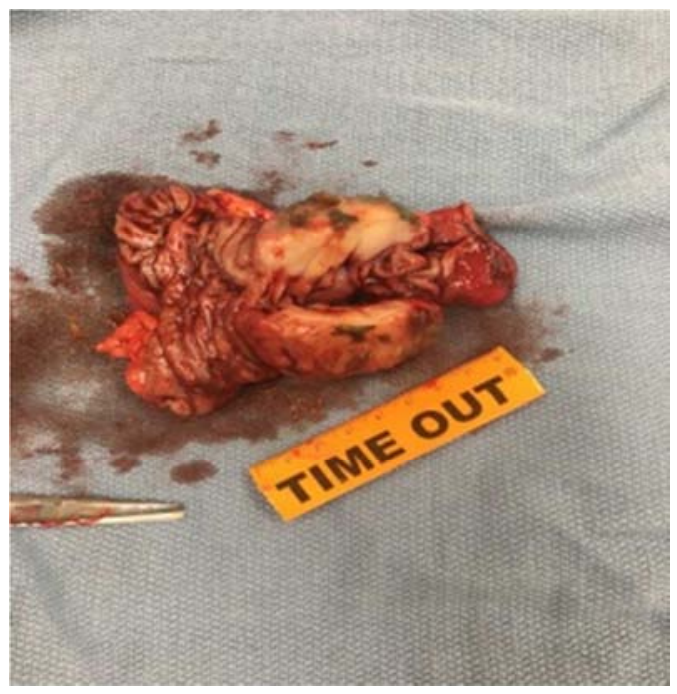

Figure 5. Gross dissection of the specimen with the cecum cut vertically along taenia coli and reflected laterally away from the tumor.

\section{Discussion}

Adult ileocecal intussusception secondary to Burkitt lymphoma is a rare clinical presentation. Adult intussusception occurs most often in the small bowel and is classified based on location. It can be categorized as enteroenteric (small bowel only), colocolic (large bowel only), ileocolic (terminal ileum prolapses within the ascending colon), or ileocecal (ileocecal valve is the lead point) [5]. Intussusception rarely occurs in adults, but nearly half of their causes are malignant [6]. $90 \%$ of intussusception occurs in children 3 months to 3 years of age with peak incidence between the fifth and ninth month of life [7, 14].

The classic triad of symptoms including abdominal pain, palpable abdominal mass and currant-jelly stool are seen only $15 \%$ of the time with pediatric presentation and are rarely seen with adult presentation. The clinical presentation of intussusception in adults can be variable, posing a challenge to diagnosis. Adult intussusceptions often present as chronic, intermittent, cramping abdominal pain associated with nonspecific signs of bowel obstruction including nausea, vomiting, gastrointestinal bleeding, constipation, or abdominal distention [5].

According to a case control study that reviewed 25 years of adult intussusception diagnoses retrospectively, an organic lesion was identified in $95 \%$ of the cases. Forty-four patients with documented intussusception were included. The mean age was 51 years (15-93 years). Fifty-eight percent of the pure colonic lesions were malignant, and $85 \%$ of them were primary adenocarcinomas [6, 15]. Primary GI lymphoma represents only $1-4 \%$ of all GI malignancies [7].

Burkitt lymphoma is a very aggressive malignancy and one of the fastest growing amongst human malignancies [12, 13]. According to the classification given by the World Health Organization, there are three types of Burkitt lymphoma based on clinical grounds; Endemic, sporadic and immunodeficiency associated. Nearly all cases are caused by EBV [12].

The endemic variant is common in Africa, the sporadic variant is present in the U.S and Western Europe, and the immunocompromised variant occurs mainly in HIV patients. The sporadic variant comprises $30 \%$ of pediatric lymphomas and less than $1 \%$ of adult Non-Hodgkin Lymphoma [12].

\section{Conclusion}

Intussusception rarely occurs in adults and typically presents with nonspecific symptoms making a preoperative diagnosis difficult. Current data supports a selective approach to operative treatment in adults. Colonic lesions should not be reduced before resection because they most likely represent a primary adenocarcinoma. Small bowel intussusception should be reduced only in patients in whom a benign diagnosis has been made preoperatively or in patients whom resection may result in short gut syndrome [11]. The likelihood of an underlying neoplasia particularly in the colon as a cause, is high. Operative management is thus almost always necessary 
[10].

CT is the most sensitive diagnostic modality and can distinguish between intussusception with and without a lead point [8]. Intussusception leads to intestinal obstruction and compromise of mesenteric blood flow with resultant inflammation and potential for ischemia [9]. It is imperative that a prompt diagnosis be made in the setting of intussusception to prevent peritonitis, bowel necrosis, perforation or other potentially fatal alternatives.

\section{References}

[1] Zhang, Jennie et al. "Carcinoid tumor causing ileoceccal intussusception in an adult patient." Journal of community hospital internal medicine perspectives vol. 9, 3 267-270. 19 Jun. 2019, doi: 10.1080/20009666.2019.1601058.

[2] Wang, Ning et al. "Adult intussusception: a retrospective review of 41 cases." World journal of gastroenterology vol. 15, 26 (2009): 3303-8. doi: 10.3748/wjg.15.3303.

[3] Wiener-Carrillo, Isidoro et al. "Intussusception secondary to a carcinoid tumor in an adult patient." International journal of surgery case reports vol. 5, 5 (2014): 265-7. doi: 10.1016/j.ijscr.2014.01.022.

[4] Azar, T, and D L Berger. "Adult intussusception.” Annals of surgery vol. 226, 2 (1997): 134-8. doi: 10.1097/00000658-199708000-00003.

[5] Lu, Teng, and Yi-mei Chng. "Adult intussusception." The Permanente journal vol. 19, 1 (2015): 79-81. doi: 10.7812/TPP/14-125.

[6] Barussaud, M., Regenet, N., Briennon, X. et al. Clinical spectrum and surgical approach of adult intussusceptions: a multicentric study. Int J Colorectal Dis 21, 834-839 (2006). https://doi.org/10.1007/s00384-005-0789-3.
[7] Bălănescu NR, Topor L, Malureanu D, Stoica I. Ileocolic intussusception due to Burkitt lymphoma: a case report. J Med Life. 2013; 6 (1): 61-64.

[8] Marinis A, Yiallourou A, Samanides L, Dafnios N, Anastasopoulos G, Vassiliou I, Theodosopoulos T. Intussusception of the bowel in adults: A review. World $\mathrm{J}$ Gastroenterol 2009; 15 (4): 407-411.

[9] Arjowan Mustafa, Lina Azzam, Hussam M. Azzam. A Case Report of a Primary Intususseption in Pregnancy. American Journal of Medical Case Reports. 2016; 4 (11): 357-360. doi: 10.12691/ajmcr-4-11-2.

[10] Begos DG, Sandor A, Modlin IM. The diagnosis and management of adult intussusception. Am J Surg. 1997 Feb; 17 3 (2): 88-94. http://dx.doi.org/10.1016/S0002-9610(96)00419-9.

[11] Eisen LK, Cunningham JD, Aufses AH., Jr Intussusception in adults: institutional review. J Am Coll Surg. 1999 Apr; 188 (4): 390-5.

DOI: http://dx.doi.org/10.1016/S1072-7515(98)00331-7.

[12] Khan, Adnan et al. "Primary Gastric Burkitt's lymphoma." Pakistan journal of medical sciences vol. 33, 5 (2017): 1294-1297. doi: 10.12669/pjms.335.13002.

[13] Erkan, Gulbanu et al. "A Burkitt's Lymphoma Case Mimicking Crohn's Disease: A Case Report." Case reports in medicine vol. 2011 (2011): 685273. doi: 10.1155/2011/685273.

[14] Seifarth FG, Triana J. Diagnosis and laparoscopic treatment of ileoileal intussusception secondary to heterotopic pancreas in an infant: case report and review of the literature. Journal of Pediatric Surgery. 2011; 46: 33-36.

[15] Zubaidi A, Al-Saif F, Silverman R. Adult intussusception: a retrospective review. Dis Colon Rectum. 2006; 49: 1546-1551. 